\title{
EL GÉNERO SICYDIUM (CUCURBITACEAE, ZANONIOIDEAE, ZANONIEAE, SICYDIINAE) EN MÉXICO
}

\author{
RAFAEL LIRA \\ Laboratorio de Recursos Naturales, Unidad de Biotecnología y Prototipos \\ Escuela Nacional de Estudios Profesionales Iztacala \\ Universidad Nacional Autónoma de México \\ Avenida de Los Barrios 1 \\ Los Reyes Iztacala, Tlalnepantla, 54090 Estado de México
}

\begin{abstract}
RESUMEN
Con base en la revisión de ejemplares de herbario y evidencias palinológicas, se presenta un estudio taxonómico de los representantes mexicanos del género Sicydium. Se reconoce un total de cuatro especies para México: S. davilae, endémica de Chiapas, S. schiedeanum y S. tamnifolium, de amplia repartición en América tropical, y S. tuerckheimii, distribuida del sur de México a Centroamérica. Se presentan comentarios de la variación encontrada en los caracteres morfológicos y palinológicos más relevantes. La parte taxonómica incluye una clave de identificación de las especies, así como descripciones, sinonimia, datos de hábitat y distribución geográfica e ilustraciones.
\end{abstract}

Palabras clave: Cucurbitaceae, México, palinología, Sicydium, sistemática.

\begin{abstract}
Based on the revision of herbarium specimens and palynological evidence, a taxonomic study of the Mexican species of the genus Sicydium is proposed. A total of four species are recognized for Mexico: Sicydium davilae, endemic to the state of Chiapas, $S$. schiedeanum and S. tamnifolium, widely distributed in tropical America, and S. tuerckheimii, distributed from southern Mexico to Central America. The paper presents comments about the variation found in the most relevant morphological and palynological characters. The taxonomic part includes an identification key to the species, as well as descriptions, synonymy, habitat and geographic distribution data, and illustrations.
\end{abstract}

Key words: Cucurbitaceae, Mexico, palynology, Sicydium, systematics. 


\section{INTRODUCCIÓN}

Sicydium es un género neotropical. Fue descrito originalmente en la primera mitad del siglo XIX por Schlechtendal (1832) con base en una planta recolectada por Schiede en el estado de Veracruz y a la cual nombró como Sicydium schiedeanum. Desde entonces se han publicado varios taxa más bajo este género; cuatro de ellos ya han sido transferidos al distante género Ibervillea (I. lindheimeri (A. Gray) Greene, I. tenuisecta (A. Gray) Small, I. tenella (Naudin) Small, I. tripartita (Naudin) Greene) (Kearns, 1994), mientras que otros siete (S. araguense Steyermark et Trujillo, S. davilae Lira, S. diffusum Cogn., S. gracile Cogn., S. schiedeanum Schltdl., S. tamnifolium (H.B.K.) Cogn. y S. tuerckheimii Donn. Sm.) se mantienen formando parte de Sicydium (Jeffrey, 1990; Lira, 1995). Los límites taxonómicos de estas siete especies se basan en el tipo, la distribución y abundancia del indumento, principalmente de sus partes vegetativas, la morfología y dimensiones de las inflorescencias y flores estaminadas, con especial atención a la forma y otros rasgos de los pétalos, la longitud de las anteras respecto a la de los filamentos y la posición de la articulación de los pedicelos florales (Cogniaux, 1881, 1916; Dieterle, 1976; Jeffrey y Trujillo, 1992; Lira, 1988, 1995; Nee, 1993; Wunderlin, 1978). En esta contribución se presentan los resultados de una revisión de las especies mexicanas de Sicydium, basada en la macromorfología y apoyada en las características de los granos de polen.

El trabajo incluye una descripción y discusión de la variación observada en los caracteres más importantes de las especies mexicanas de Sicydium, así como también el estudio taxonómico del género. A falta de otras evidencias, el concepto de especie utilizado aquí se basa fundamentalmente en datos morfológicos.

\section{MATERIALES EXAMINADOS}

Se consultaron 373 ejemplares de herbario correspondientes a 208 números de colecta (incluyendo los tipos nomenclaturales y colectas del autor), depositados en 17 herbarios; de los Estados Unidos (F, GH, MICH, MO, NY, US, TEX-LL), de México (CHAPA, ENCB, IEB, MEXU, XAL), de América del Sur (COL) y de Europa (BM, BR, K, P).

\section{RESULTADOS}

Diversidad y distribución de las especies de Sicydium en México

El género Sicydium está representado en México por cuatro especies: S. tamnifolium (H.B.K.) Cogn., S. schiedeanum Schltdl., S. davilae Lira 
y S. tuerckheimii Donn. Sm. (Lira, 1995; Lira et al., 2002); su distribución se extiende desde Tamaulipas hasta la Península de Yucatán y Chiapas (Fig. 1). Tiene la particularidad de estar ausente en el occidente del país, en donde crece el otro miembro mexicano de la subfamilia Zanonioideae, Chalema synanthera Dieterle, género y especie endémicos de México. La mayoría de los miembros de Sicydium prosperan entre el nivel del mar y los $1800 \mathrm{~m}$, y sólo $S$. schiedeanum ha sido recolectada por arriba de los $2000 \mathrm{~m}$. En los sitios en donde es más probable encontrarlas prevalecen suelos calcáreos y ricos en materia orgánica; S. tamnifolium y $S$. tuerckheimii tienen la capacidad de prosperar también en terrenos inundables. La vegetación primaria de los ambientes en que crecen las especies de Sicydium corresponde a bosques tropicales caducifolios, perennifolios, subperennifolios y mesófilos de montaña, aunque también es muy frecuente encontrarlas en comunidades secundarias derivadas de los anteriores, así como en potreros y otros sitios abiertos. En México cuando menos tres de las especies pueden coexistir en una misma zona y ello sugiere la posibilidad de cruzamientos interespecíficos. De hecho, la rareza y la originalidad, sobre todo palinológica, de una especie recientemente descrita, Sicydium davilae Lira (Lira, 1995; Lira et al., 1998), indican que pudiera tratarse de un híbrido entre $S$. schiedeanum y $S$. tamnifolium, plantas que también se han recolectado en el municipio de Ocozocuautla, Chiapas, en donde se ubica la localidad tipo de $S$. davilae.

\section{Hábito y Características Vegetativas}

Las especies de Sicydium son hierbas rastreras a trepadoras, dioicas, con tallos delgados y raíces perennes de color rojizo, delgadas y más o menos ramificadas. Las láminas foliares son enteras a ligeramente lobuladas o al menos angulosas (Fig. 5) y, al igual que los tallos, pueden ser desde glabras hasta densamente pubescentes o tomentosas (Fig. 2). El indumento está constituido por pelos multicelulares, uniseriados, glandulares y no glandulares. Aunque en general ambos tipos de tricomas se encuentran mezclados en los tallos y hojas de la mayoría de las especies, en Sicydium davilae los primeros aparentemente están ausentes o son muy escasos e inconspicuos.

La distribución y densidad de la pubescencia en las partes vegetativas en ocasiones también permite reconocer algunas especies. Así, Sicydium schiedeanum se distingue por presentar hojas y tallos esparcidamente puberulentos a totalmente glabros. Por su parte, S. tuerckheimii tiene hojas cuya superficie adaxial es escabrosa y áspera al tacto, mientras la abaxial es densamente pubescente a tomentosa, y sus tallos pueden ser desde puberulentos hasta densamente pubescentes. La especie más distintiva en cuanto a estos rasgos es $S$. davilae, cuyos tallos y hojas son suaves al tacto y densamente pubescentes a tomentosos o lanados. 
Acta Botanica Mexicana 68: 39-64 (2004)
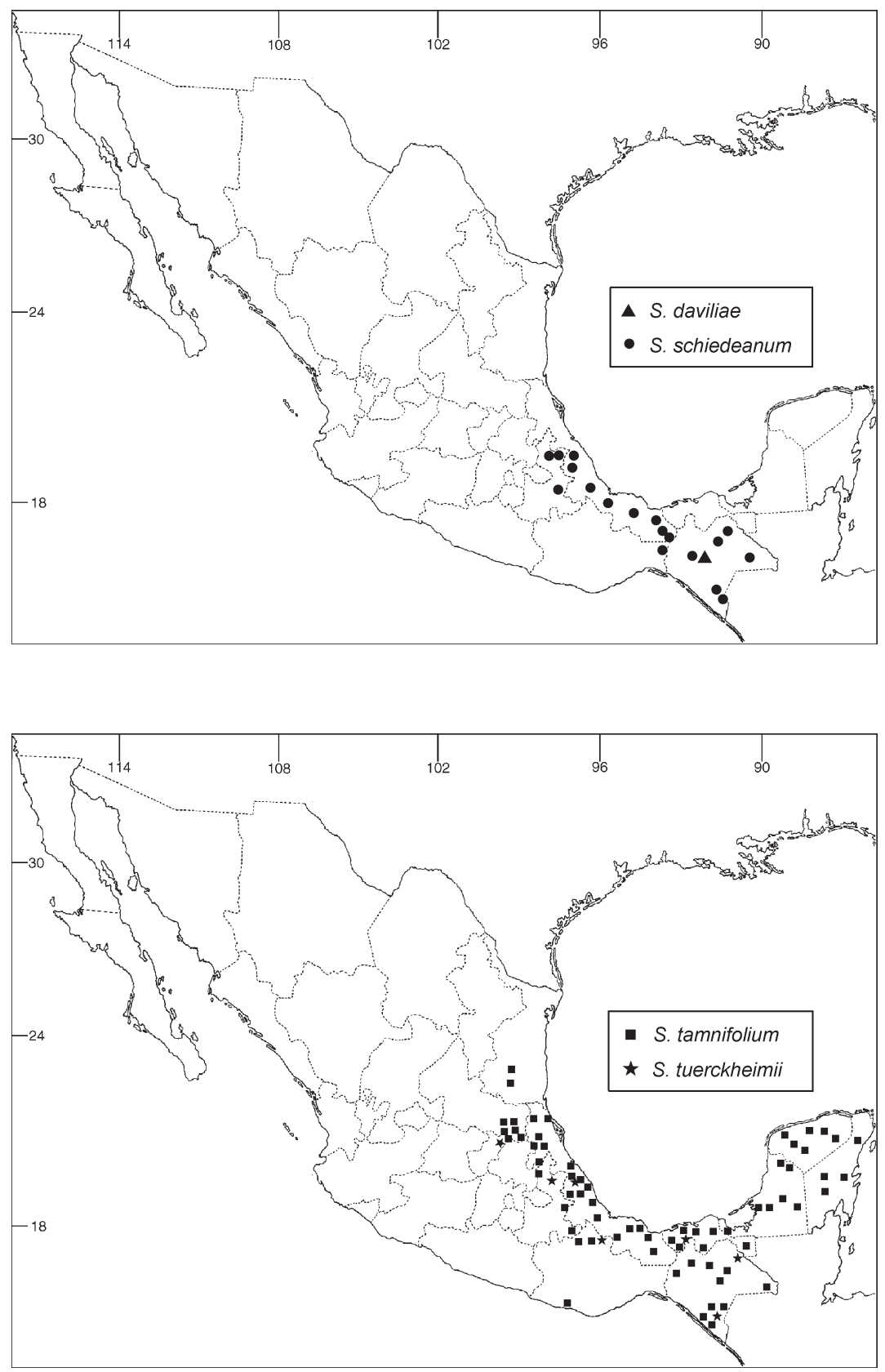

Fig. 1. Distribución geográfica de las especies mexicanas de Sicydium. 


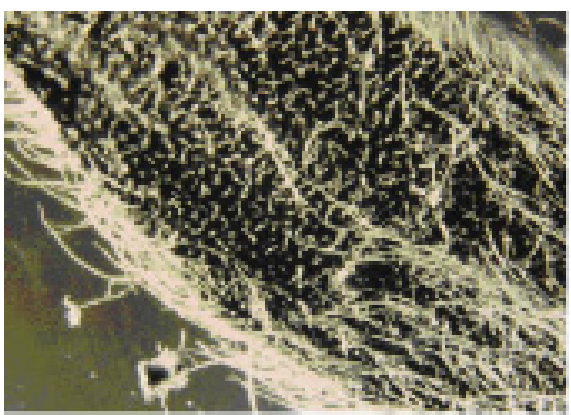

a

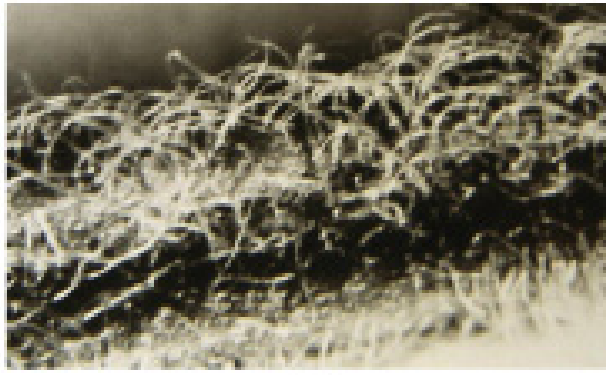

b

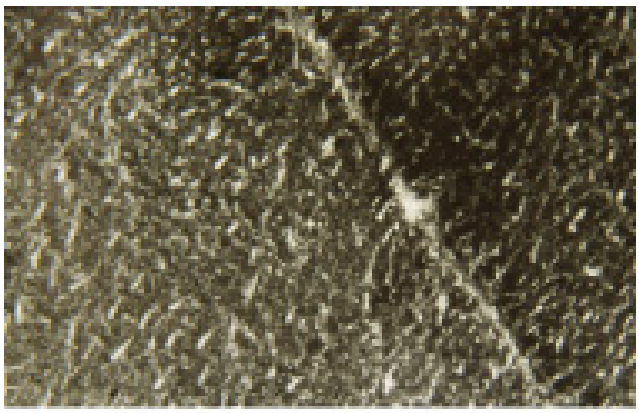

$\mathrm{c}$

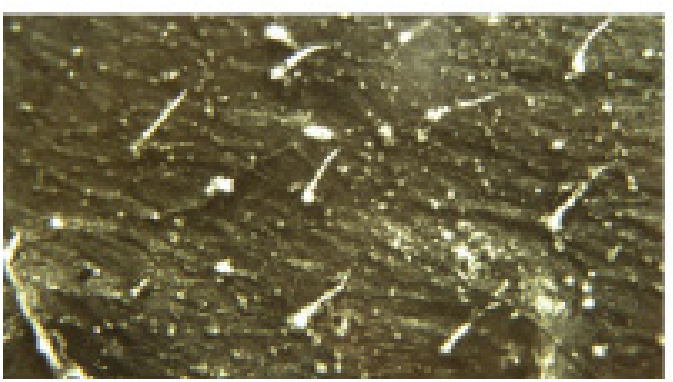

$\mathrm{e}$

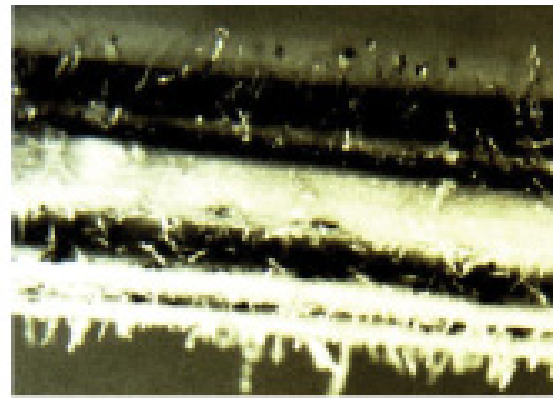

d

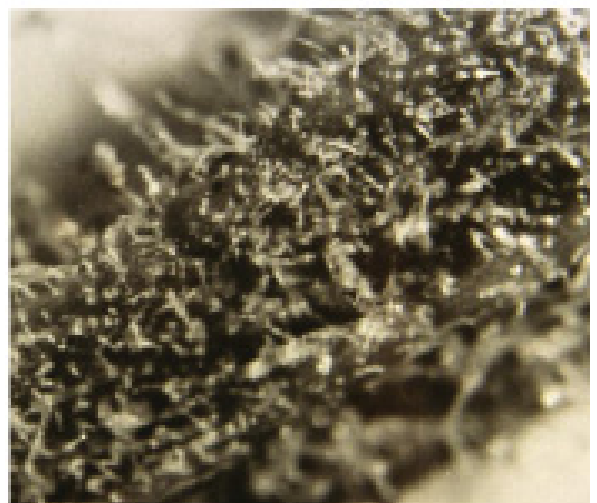

f

Fig. 2. Pubescencia de hojas y tallos en algunas especies mexicanas de Sicydium. a-b) $S$. davilae, c-d) S. tamnifolium, e-f) S. tuerckheimii. 
En S. tamnifolium se presenta la mayor variación en tales características, pues es posible encontrar poblaciones con hojas y tallos cuyo indumento puede ser desde densa y suavemente pubescente, hasta otras en donde es esparcida y diminutamente puberulento e incluso algo escabroso.

La coloración que adquieren las hojas y los tallos al secar, en algunos casos también puede ser utilizada como un elemento auxiliar para el reconocimiento de las especies, especialmente cuando sólo se cuenta con plantas femeninas. Así por ejemplo, en Sicydium schiedeanum, y sobre todo en $S$. tuerckheimii, las hojas frecuentemente presentan tonalidades pardusco-rojizas, mientras que en $S$. davilae y $S$. tamnifolium comúnmente son verdes a verde-amarillentas o blanquecinas.

\section{Características florales}

Indudablemente los principales caracteres de valor taxonómico en Sicydium se encuentran en las flores estaminadas. En contraste, en las pistiladas hasta la fecha no se ha detectado ninguno de gran importancia, lo cual puede atribuirse a que las plantas femeninas son las menos representadas en materiales de herbario. Así, por ejemplo, en este trabajo se dan a conocer por primera vez estas flores para $S$. tuerckheimii, aunque con base en un solo ejemplar procedente de Nicaragua. Las características más importantes de las flores estaminadas y pistiladas de las especies de Sicydium se describen a continuación y se ilustran las estaminadas de dos de ellas en la figura 3.

Características y disposición de las flores. Las especies de Sicydium son dioicas, rasgo que las hace diferentes de su pariente mexicano Chalema synanthera; sus flores son diminutas, pentámeras y están dispuestas en panículas muy ramificadas, en cuyas axilas se desarrollan brácteas u hojas reducidas. Estas brácteas son de distintos tamaños y pueden ser desde lanceoladas y sésiles, hasta ovadocordadas y cortamente pecioladas. Las panículas estaminadas son frecuentemente de mayores dimensiones y más apretadas que las pistiladas y las brácteas son, en general, mucho más abundantes y conspicuas que en las inflorescencias pistiladas. Las flores llevan pedicelos solitarios o más comúnmente agrupados en fascículos, racimos o agrupaciones subumbeliformes. En términos generales, las inflorescencias estaminadas de $S$. schiedeanum y sobre todo de $S$. tuerckheimii, son más grandes que las de $S$. tamnifolium y $S$. davilae, aunque en el caso de la última especie se cuenta con muy poca información acerca de la variación en todas sus características, pues ésta sólo se conoce del ejemplar tipo.

Pedicelos. Los pedicelos de las flores estaminadas son delgados y presentan un ligero engrosamiento (articulación) en un punto del mismo, cuya ubicación es un carácter diagnóstico importante para separar dos grupos dentro de las especies 


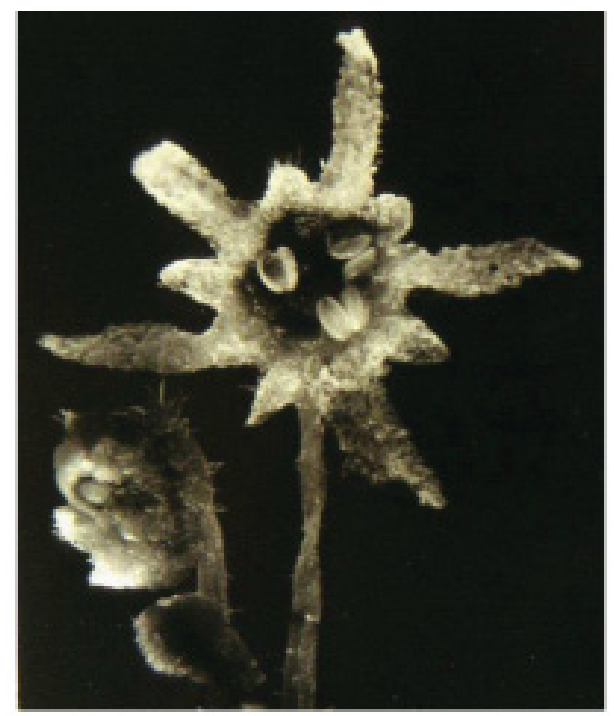

a

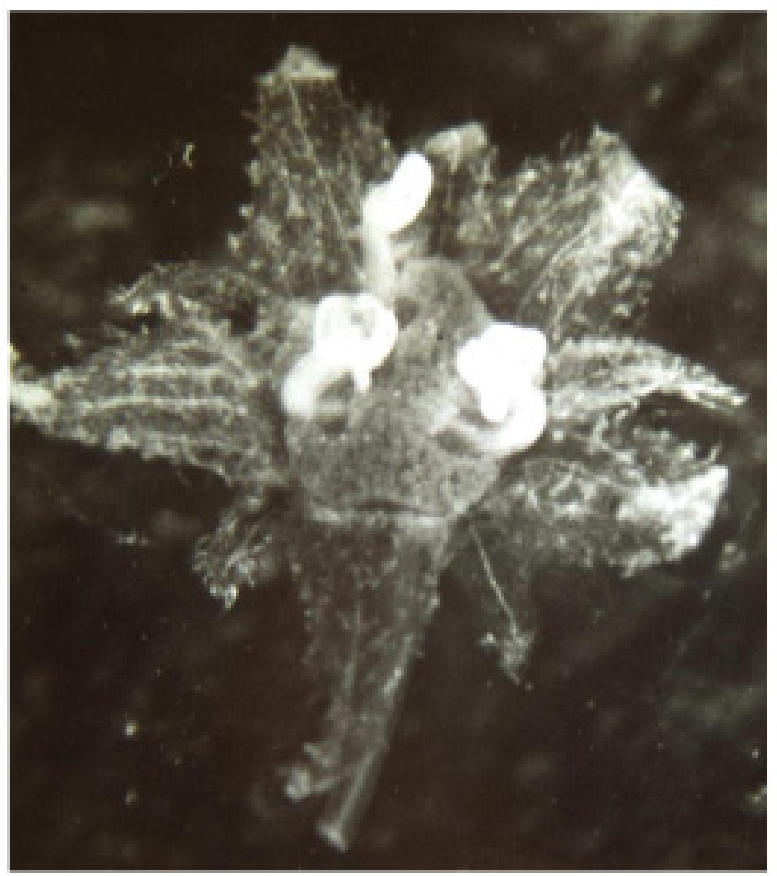

b

Fig. 3. Flores estaminadas de (a) Sicydium tamnifolium y (b) S. tuerckheimii (40 X). 
mexicanas. Así, mientras en S. tamnifolium la articulación está ubicada hacia la base, en las restantes especies se presenta en la porción media o poco más arriba. Los pedicelos de las flores pistiladas son más robustos (mucho más en los frutos), no están articulados y en algunos materiales de S. tamnifolium los pelos que los cubren son sumamente abundantes y patentes. Esto se aplica a todas las especies, excepto S. davilae cuyas plantas femeninas aún se desconocen (Lira, 1995).

Perianto. El receptáculo es pateliforme, glabro a pubescente y está muy reducido, como ocurre en muchos grupos de la familia Cucurbitaceae; los sépalos son triangulares a lanceolados y generalmente tienen la mitad (o menos) de la longitud de los pétalos; estos últimos son de color blanco-verdoso o crema-verdoso y pueden ser ovado-triangulares a lanceolados, con los bordes enteros a erosos o fimbriados.

Estambres. Los estambres son 3, completamente libres (otra característica que separa a Sicydium de Chalema) y están insertos cerca de la periferia de la base del receptáculo. Las anteras son oblongas, dos bitecas y una monoteca, con las tecas rectas que se abren por una sutura longitudinal; el conectivo puede estar sumamente reducido o prácticamente ausente como en $S$. tamnifolium, o ser muy conspicuo y agrandado como en las restantes especies. Como ya se ha señalado, un carácter de relevancia taxonómica es la proporción entre el tamaño de las anteras y los filamentos. Por ejemplo, en la mayoría de los materiales de S. tamnifolium las anteras son prácticamente sésiles, pero se pueden observar otros ejemplares en donde los filamentos son tan largos como las anteras. En contraste, en el resto de las especies los filamentos son 1-2 veces más largos que las anteras. En este aspecto la revisión de los ejemplares tipo reveló ciertas inconsistencias respecto al contenido de las descripciones originales correspondientes. Así, mientras que en las descripciones de $S$. coriaceum, S. glabrum, S. schiedeanum y $S$. tuerckheimii se indica que las anteras y los filamentos tienen la misma longitud (Cogniaux, 1881; Donell-Smith, 1911; Schlechtendal, 1832; Standley y Steyermark, 1944), en los ejemplares tipo correspondientes se encontró que los filamentos son 1-2 veces más largos que las anteras. Esta fue una de las características más importantes que se tomó en cuenta para considerar a $S$. coriaceum y $S$. glabrum como sinónimos de $S$. schiedeanum.

Ovario, estilos y estigmas. El ovario es unilocular, con un sólo óvulo péndulo y por fuera puede ser esparcida a densamente pubescente a puberulento o incluso casi glabro. Los estilos y estigmas son 3 , lineares y están insertos cerca de la periferia de la base del receptáculo. Tres estaminodios anteriformes generalmente están presentes en la base del receptáculo de la flor pistilada. En algunos ejemplares de S. tamnifolium se observó que los estaminodios pueden tener el aspecto de estambres normales. 
Frutos y semillas

Los frutos son globosos y generalmente presentan restos del perianto hasta etapas avanzadas de su desarrollo (Fig. 4). Miden entre 4 y $12 \mathrm{~mm}$ de diámetro y cuando jóvenes son de color verde, tornándose purpúreos obscuros a negros al madurar. El epicarpo es delgado y liso y el mesocarpo carnoso-jugoso y de sabor más o menos amargo. La semilla es globosa, tiene el hilo relativamente prominente y la testa es rígida y fuertemente crestada. Aunque en apariencia no parece haber diferencias entre las especies en la estructura y disposición de las crestas de la semilla, valdría la pena hacer un estudio más preciso de esta estructura para tratar de buscar caracteres adicionales de valor diagnóstico a nivel de especie.

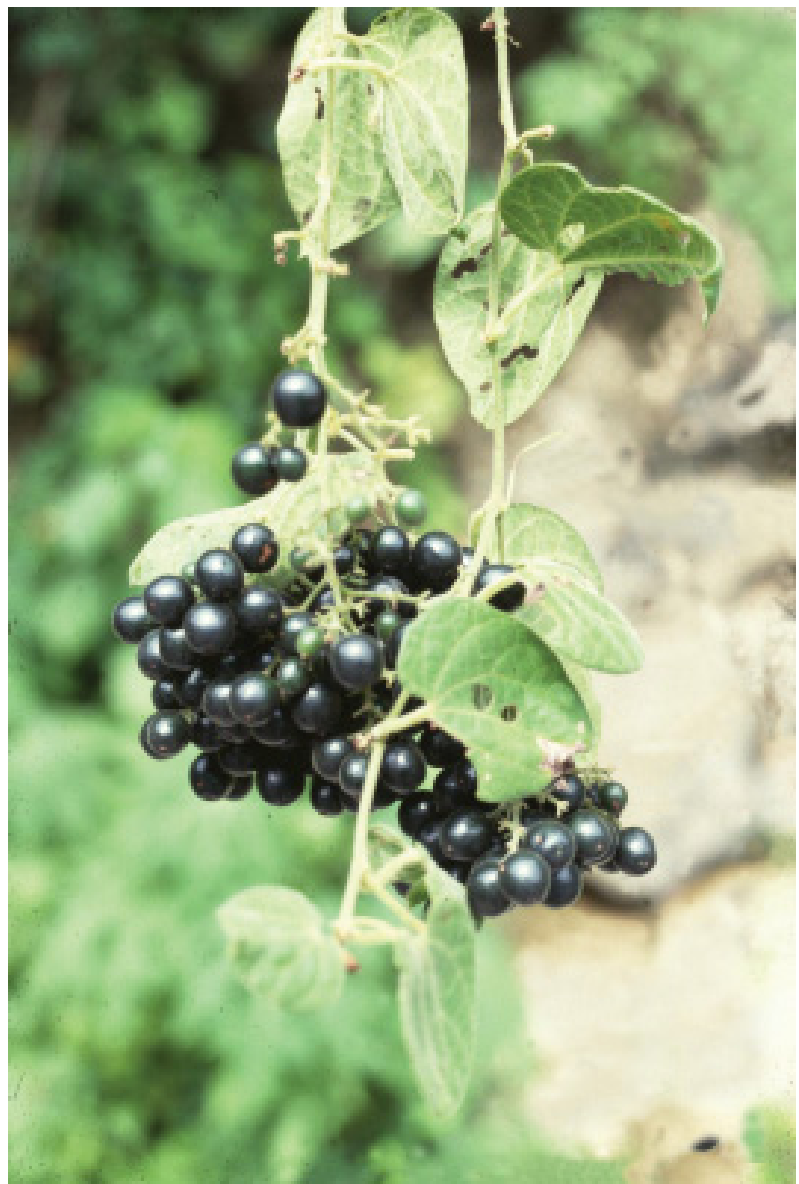

Fig. 4. Frutos de Sicydium tamnifolium. 
Polen

Como ocurre en la entera subfamilia Zanonioideae, los granos de polen en todas las especies de Sicydium son comparativamente pequeños, estriados y con las estrías poco ramificadas y anastomosadas. No obstante, los datos cuantitativos (Lira et al., 1998) han revelado algunas diferencias interesantes entre las especies mexicanas (Cuadro 1). Por ejemplo, Sicydium tuerckheimii presenta los granos más grandes, en algunos casos con medidas en el eje ecuatorial de hasta ca. $35 \mu \mathrm{m}$. En contraste, los de menor tamaño y con menor distancia entre colpos $(4.1 \mu \mathrm{m})$ son los de Sicydium davilae, además de ser los únicos de forma esferoidal u oblatoesferoidal. Por su parte, Sicydium tamnifolium se distingue de las restantes especies del género porque en sus granos se encontró la mayor distancia entre colpos $(7.5 \mu \mathrm{m})$.

Parte Taxonómica

Sicydium Schlechtendal, Linnaea 7: 388. 1832, non A. Gray 1850. Tipo: $S$. schiedeanum.

Plantas herbáceas, rastreras a trepadoras vigorosas, perennes, dioicas; raíces delgadas, poco ramificadas, rojizas; tallos sulcados, ramificados, casi glabros a diversamente indumentados; hojas pecioladas, las láminas foliares ovado-cordadas a ovado-lanceoladas, enteras a ocasionalmente angulosas o lobuladas; zarcillos simples a bifurcados hacia el ápice, enrollándose por arriba y por abajo del punto de ramificación; flores diminutas, las estaminadas dispuestas en panículas apretadas, pedunculadas, usualmente con hojas reducidas o brácteas en la base del pedúnculo de la inflorescencia, de los pedicelos, y en las axilas de las ramificaciones de la inflorescencia, pedicelos solitarios, con más frecuencia muy densamente aglomerados en fascículos o agrupaciones subumbeliformes, delgados, con un abultamiento o articulación hacia la base, en la porción media o poco más arriba, perianto 5-mero, receptáculo pateliforme, sépalos patentes, corola crema-verdosa o blanco-verdosa, profundamente 5-dividida casi hasta la base, pétalos triangulares a lanceolados, patentes, enteros a ligeramente eroso-fimbriados, estambres 3, completamente libres, insertos en o cerca de la base del receptáculo, filamentos desde casi nulos hasta 1 a 2 veces más grandes que las anteras, anteras oblongas, dos bitecas y una monoteca, las tecas rectas, abriendo por una sutura longitudinal, el conectivo desde prácticamente nulo hasta más o menos ensanchado, polen estriado, 3-4-colporado; flores pistiladas dispuestas como las estaminadas, aunque las inflorescencias mucho menos apretadas, pedicelos angulosos, más gruesos que los de las estaminadas y no articulados, perianto como en las estaminadas pero el receptáculo muy reducido a obsoleto, ovario ovoide, unilocular, óvulo 1, péndulo, estilos 3 , lineares, estigmas 
Lira: El género Sycidium en México

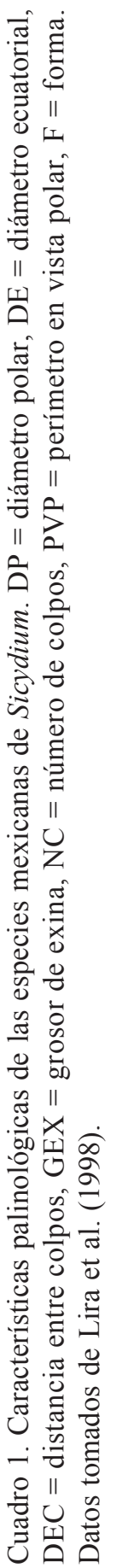

\begin{tabular}{|c|c|c|c|c|}
\hline I & 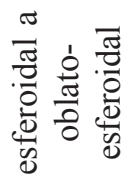 & 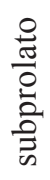 & 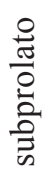 & $\begin{array}{l}\text { 总 } \\
0 \\
0 \\
0 \\
0 \\
0\end{array}$ \\
\hline \& & $\frac{\text { పี }}{\stackrel{0}{*}}$ & 总 & 苛 & 总 \\
\hline Z & $\begin{array}{l}+ \\
1 \\
m\end{array}$ & $m$ & $m$ & $n$ \\
\hline 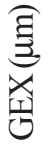 & $\begin{array}{l}0 \\
\text { v } \\
v\end{array}$ & $\stackrel{\circ}{\stackrel{\sim}{v}}$ & $\stackrel{\stackrel{o}{i}}{\stackrel{v}{v}}$ & $\begin{array}{l}0 \\
\text { v } \\
v\end{array}$ \\
\hline 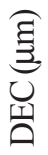 & F & $\stackrel{\circ}{\circ}$ & $\stackrel{n}{r}$ & $\begin{array}{l}o \\
i\end{array}$ \\
\hline 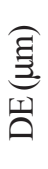 & $\begin{array}{l}-7 \\
\\
1 \\
b \\
\vdots \\
=\end{array}$ & $\begin{array}{l}\stackrel{\sim}{+} \\
\underset{\sim}{1} \\
1 \\
\infty \\
\infty\end{array}$ & $\begin{array}{l}0 \\
\stackrel{\sim}{N} \\
1 \\
\infty \\
\dot{0}\end{array}$ & 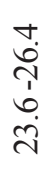 \\
\hline 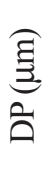 & $\begin{array}{l}0 \\
\stackrel{n}{7} \\
1 \\
n \\
=\end{array}$ & $\begin{array}{l}\infty \\
\stackrel{\infty}{a} \\
1 \\
\\
\end{array}$ & 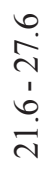 & $\begin{array}{l}0 \\
\dot{\sim} \\
m \\
\dot{+} \\
\dot{0} \\
\sim\end{array}$ \\
\hline 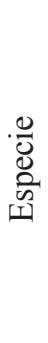 & 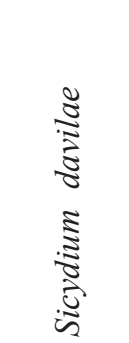 & 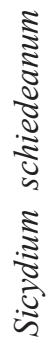 & 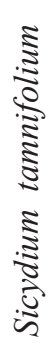 & 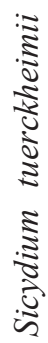 \\
\hline
\end{tabular}


3, lineares, patentes, estaminodios 3 , usualmente presentes, en algunos casos con aspecto de estambres normales; frutos globosos, indehiscentes, epicarpo delgado, coriáceo, verde cuando joven, tornándose de color púrpura obscuro o negro al madurar, mesocarpo jugoso-carnoso; semilla esférica, rostrada, fuertemente ornamentada con abundantes crestas angulosas de diferentes tamaños.

Clave de identificación de las especies

1. Pedicelos de las flores estaminadas articulados en la porción media o poco más arriba; filamentos 1 a 2 veces más largos que las anteras; conectivo comparativamente ensanchado; láminas foliares glabras a tomentosas o lanadas al menos en la superficie abaxial.

2. Plantas glabras o sólo ligeramente puberulentas, los pelos usualmente de menos de $1 \mathrm{~mm}$ de largo; láminas foliares siempre enteras, frecuentemente lustrosas en ambas superficies

S. schiedeanum

2. Plantas por lo general conspicuamente puberulentas, pubescentes, tomentosas o algunas veces incluso lanadas al menos en la superficie abaxial de las láminas foliares, los pelos desde ca. $1 \mathrm{~mm}$ hasta de $3 \mathrm{~mm}$ de largo y fácilmente visibles; láminas foliares enteras, angulosas o ligeramente lobadas o lobuladas, no lustrosas.

3. Tallos y láminas foliares densamente tomentosas a lanadas, el indumento básicamente de pelos no glandulares y de color blanquecino a amarillento al secar S. davilae

3. Tallos puberulentos a conspicuamente pubescentes, las láminas foliares puberulentas y áspero-escabrosas en la superficie adaxial, la abaxial suavemente pubescente a tomentosa, el indumento de tricomas glandulares y no glandulares y usualmente de coloración pardo obscuro rojiza o algunas veces verdosa al secar

S. tuerckheimii

1. Pedicelos de las flores estaminadas articulados hacia la base; filamentos nulos a casi tan largos como las anteras; conectivo nulo o muy escasamente desarrollado; láminas foliares puberulentas a densamente pubescentes (rara vez casi glabras) en ambas superficies

S. tamnifolium

Sicydium davilae Lira, Novon 5: 286. 1995. "daviliae” Tipo: México, Chiapas, Ocozocuautla, camino Horizonte a Santa Laura, F. Vázquez B. 1024 (holotipo XAL!).

Plantas herbáceas, trepadoras, densamente pubescentes a tomentosas o lanadas, indumentadas con pelos suaves, septados, no glandulares, de hasta $3 \mathrm{~mm}$ 
de largo, presentando tonalidades amarillentas o blanquecinas al secar; tallos delgados, sulcados; láminas foliares de $3.5-5 \mathrm{~cm}$ de largo y $3-5 \mathrm{~cm}$ de ancho, ovado-cordadas, enteras a 3-anguloso-lobadas, el seno basal cordado a rectangular, los bordes enteros, el ápice agudo a acuminado, de consistencia subcoriácea, amarillentas o blanquecinas al secar, ambas superficies densamente pubescentes a tomentosas o lanadas, pecíolos de 1 a $2 \mathrm{~cm}$ de largo, sulcados, indumentados como el tallo; zarcillos densamente pubescentes a tomentosos o lanados; panículas estaminadas de 4 a $6 \mathrm{~cm}$ de largo, densamente pubescentes; brácteas unas cuantas, ovado-cordadas a ovado-lanceoladas, pedicelos de 1 a $1.3 \mathrm{~mm}$ de largo, filiformes, pubescentes, articulados a la mitad o poco más arriba, receptáculo de $0.2 \mathrm{~mm}$ de largo y 1 a $1.2 \mathrm{~mm}$ de ancho, puberulento, sépalos de 0.5 a $0.6 \mathrm{~mm}$ de largo y $0.3 \mathrm{~mm}$ de ancho, triangulares, puberulentos, pétalos de 1 a $1.3 \mathrm{~mm}$ de largo y $0.5-0.6 \mathrm{~mm}$ de ancho, ovado-lanceolados, puberulentos, los bordes enteros, el ápice acuminado, filamentos de 0.4 a $0.6 \mathrm{~mm}$ de largo, anteras de 0.2 a $0.3 \mathrm{~mm}$ de largo, el conectivo comparativamente ensanchado y ligeramente bifurcado en el ápice; flores pistiladas $\mathrm{y}$ frutos desconocidos.

Distribución y hábitat. Endémica del estado de Chiapas, México y sólo conocida de la colección tipo. Crece en bosque tropical perennifolio perturbado a 900 m s.n.m.

Notas. De $S$. davilae sólo se conocen las plantas masculinas representadas en la colección tipo. Sin embargo, de acuerdo con la información de la etiqueta de tal ejemplar, es probable que algunos de sus duplicados correspondan a individuos femeninos con frutos. Además de las características macromorfológicas que distinguen a este taxon, los rasgos de sus granos de polen también son distintivos dentro del género. Así, mientras que en todas las especies estudiadas los granos de polen son principalmente tricolporados y prolatos a subprolatos, en $S$. davilae se han encontrado, además, granos tri o tetracolporados y de forma oblato-esferoidal. Como ya se señaló, la existencia de poblaciones de $S$. schiedeanum y $S$. tamnifolium en la región de la localidad tipo de esta especie, aunada a sus rasgos palinológicos un tanto anormales, sugieren que se trata de un taxon de origen híbrido. Esto último, sin embargo, debe ser confirmado, pues los cruzamientos interespecíficos exitosos entre estas dos especies no parecen ser comunes, ya que en otros sitios en donde también coexisten no se han documentado individuos o poblaciones con características que así lo sugieran.

Ejemplares examinados. CHIAPAS: mpio. Ocozocuautla de Espinoza. Camino Horizonte a Santa Laura, F. Vázquez B. 1024 (XAL). 
Sicydium schiedeanum Schltdl., Linnaea 7: 388. 1832. Tipo: México, Veracruz, Hacienda de La Laguna, C. J. W. Schiede 51 (holotipo B; isotipos P!, F!, fragmento, MO!; fotos, ENCB!, MICH!,). Sicydium coriaceum Cogn., DC. Monogr. Phan. 3: 904. 1881.Tipo: Colombia, Nova Granata ad La Mesa et Rio Magdalena, Prov. Bogotá. J. J. Triana 3024 (holotipo P!; isotipo F!, fragmento; fotos GH!, MICH!). Sicydium glabrum Standl. \& Steyerm., Field Mus. Bot. Ser. 23: 95.1944. Tipo: Guatemala, Quetzaltenango, Montaña Chicharro, lower southeastern slopes of Volcán de Santa María, 1400-1500 m. January, 17, 1940. J. A. Steyermark 34273 (holotipo F!; foto MICH!).

Plantas herbáceas, trepadoras, glabras a esparcida y diminutamente puberulentas, los pelos por lo general de menos de $1 \mathrm{~mm}$ de largo; tallos delgados, sulcados; láminas foliares de 4 a $5 \mathrm{~cm}$ de largo y 3.5 a $9 \mathrm{~cm}$ de ancho, anchamente ovado-cordadas a ovado-lanceoladas, enteras, el seno cordado a rectangular, los bordes enteros, el ápice agudo a acuminado, de consistencia herbáceo-membranácea a subcoriácea, usualmente lustrosas y verdes o algunas veces pardo obscuro-rojizas al secar, ambas superficies casi glabras a esparcida y diminutamente puberulentas, pecíolos de 1 a $4 \mathrm{~cm}$ de largo, delgados, sulcados, esparcidamente puberulentos; zarcillos esparcida y diminutamente puberulentos; panículas estaminadas de (7-) 8-15 (-20) cm de largo, esparcida y diminutamente puberulentas a más comúnmente casi glabras, las brácteas ovado-lanceoladas a lanceoladas, puberulentas, pedicelos de 1 a $3 \mathrm{~mm}$ de largo, filiformes, puberulentos, glabrescentes, articulados poco más arriba de la mitad o hacia el tercio superior, receptáculo de 0.3 a $0.4 \mathrm{~mm}$ de largo y 0.4 a $0.5 \mathrm{~mm}$ de ancho, puberulento, sépalos de 0.5 a $0.8 \mathrm{~mm}$ de largo, lanceolados, agudos, puberulentos, pétalos de 1.5 a $2.5 \mathrm{~mm}$ de largo y 1 a $1.5 \mathrm{~mm}$ de ancho, ovadotriangulares a lanceolados, acuminados, casi glabros a esparcidamente puberulentos, los bordes enteros a ligeramente erosos, filamentos de 0.6 a $0.7 \mathrm{~mm}$ de largo, anteras de 0.2 a $0.3 \mathrm{~mm}$ de largo, el conectivo comparativamente ensanchado y algo bifurcado en el ápice; panículas pistiladas de 5 a $15(-20) \mathrm{cm}$ de largo, pedicelos florales de 1.5 a $3 \mathrm{~mm}$ de largo, robustos, puberulentos, ovario de $1.5 \mathrm{~mm}$ de diámetro, globoso, puberulento a glabro, estilos y estigmas como en el género; frutos de 7 a $12 \mathrm{~mm}$ de diámetro, globosos, puberulentos, glabrescentes, pedúnculos de 2 a 4 (-6) $\mathrm{mm}$ de largo, robustos, puberulentos; semilla de 6 a $9 \mathrm{~mm}$ de diámetro.

Distribución y hábitat. De México al norte de América del Sur (Colombia y Venezuela). Crece en zonas con elevaciones entre 100 y 2540 m s.n.m., en bosques tropicales perennifolios y subperennifolios primarios y secundarios con Dialium, Bursera, Pseudobombax, Brosimum, etc., y en bosques mesófilos de montaña con Quercus, Persea, Nectandra, Mirandaceltis, Turpinia, Ulmus, Alnus y Calatola. 
También puede encontrarse en acahuales con Bursera, Schizolobium, Cassia, Luehea, Hampea, Ficus y Cnidoscolus.

Nombres comunes. En Veracruz se le conoce como "uva amarga", mientras que en otros países como Guatemala recibe los nombres "hoja de esperanza", "murciélago triángulo" y "pashté de montaña".

Notas. La revisión de los tipos de S. coriaceum y S. glabrum, reveló que en ambos casos los filamentos son 1-2 veces más largos que las anteras. La combinación de este rasgo con la esparcida y diminuta pubescencia de las láminas foliares y otras partes vegetativas, así como la posición en la porción media o poco más arriba de la articulación de los pedicelos florales, fue lo que se consideró para ubicar a ambos nombres como sinónimos de $S$. schiedeanum.

Ejemplares examinados. CHIAPAS: Mpio. Berriozábal. $13 \mathrm{~km} \mathrm{~N}$ of Berriozábal near Pozo Turipache and Finca El Suspiro, D. E. Breedlove 30794 (MEXU). Mpio. Motozintla de Mendoza. Boquerón, $15^{\circ} 15^{\prime}$ N, $92^{\circ} 17^{\prime}$ W, E. Matuda 5349 (MEXU, TEX-LL). Mpio. Ocosingo. $7 \mathrm{~km}$ al S de la desviación a Chancala, sobre la carretera a Ocosingo, E. Cabrera y H. de Cabrera 6221 (MEXU); $12 \mathrm{~km}$ al S de Palenque ó $13 \mathrm{~km}$ al S de la desviación a Chancala, sobre la carretera a Ocosingo, $17^{\circ} 23^{\prime} \mathrm{N}, 91^{\circ} 52^{\prime} \mathrm{W}$, E. Cabrera y H. de Cabrera 12346 (MEXU); $1 \mathrm{~km}$ al S de El Piedrón, camino a Chancala, E. Martínez y W. D. Stevens 24013, (MEXU). Mpio. Ocozocoautla de Espinoza. $20 \mathrm{~km} \mathrm{~N}$ of Ocozocoautla de Espinoza along road to Mal Paso, 165' N, 93²6' W, D. E. Breedlove 20990 (ENCB, MEXU, NY). Mpio. Palenque. 6-12 km S of Palenque on the road to Ocosingo, $17^{\circ} 27^{\prime} \mathrm{N}, 61^{\circ} 57^{\prime} \mathrm{W}$, D. E. Breedlove 26558 (MEXU, TEX-LL), 28823 (MEXU). Mpio. Unión Juárez. Tacaná, $15^{\circ} 08^{\prime}$ N, 9207' W, E. Matuda 18220 (MEXU). Mpio. Yajalón. San Luis, A. Shilom Ton 4531 (MEXU); cerrito Tizon, A. Shilom Ton 6735 (MEXU). OAXACA: Mpio. Santa María Chimalapa. $3 \mathrm{~km}$ al N de Santa María, bajada de Tzeuyuatza, 1655' N, 9441' W, H. Hernández 1365 (MEXU). PUEBLA: Mpio. Ahuacatlán. $4.5 \mathrm{~km}$ al SE de Ahuacatlán, brecha a Zapotitlán, $20^{\circ} 00^{\prime} \mathrm{N}, 97^{\circ} 49^{\prime} \mathrm{W}$, P. Tenorio y C. Romero 11422 (MEXU). Mpio. Mazatepec. Las Amacas, $1 \mathrm{~km}$ al W de Mazatepec, brecha a Cuetzalan, $20^{\circ} 06^{\prime} \mathrm{N}, 97^{\circ} 31^{\prime} \mathrm{W}$, P. Tenorio 15831 (MEXU). Mpio. Yaonahuac. Atotocoya, $20 \mathrm{~km}$ al $\mathrm{S}$ de Mazatepec, 1953'00" N, 97²7'00" W, A. Campos, A. Toriz y P. Tenorio 597, 598, (MEXU). VERACRUZ: Mpio. Acajete. Plan de Cedeño, F. Ventura A. 3675, 00/5/1972. (MICH). Mpio. Catemaco. $8 \mathrm{~km}$ al SE de El Bastonal, $18^{\circ} 15^{\prime} \mathrm{N}$, 95 $02^{\prime}$ W, R. Cedillo 3420 (MEXU); Bastonal-Sierra Santa Martha road, ca. $14 \mathrm{~km}$ E of Lago Catemaco, 18²5' N, 94'59' W, A. Gentry et al. 32424, 32436, 29/5/1981, (MO, XAL); camino Bastonal a Santa Martha, 18²4' N, 9457' W, A. Gómez- 
Pompa et al. 5472 (XAL); along dirt road 3.5-5.5 km E of Tebanca (3.5-5.5 km $\mathrm{E}$ of east side of Lago Catemaco, $18^{\circ} 24^{\prime} \mathrm{N}, 95^{\circ} 00^{\prime} \mathrm{W}$, B. F. Hansen y M. Nee 7618, 7628 (F, MO, XAL). Mpio. Hidalgotitán. km 1-6 del camino Cedillo-La

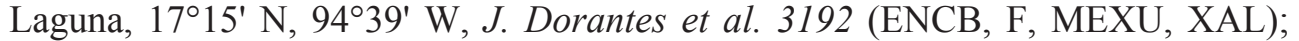
km 0-6 del camino Cedillo-Miguel Alemán, $17^{\circ} 15^{\prime}$ N, 944' W, J. Dorantes 3513

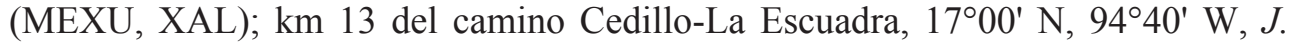
Dorantes 3709 (MEXU, MO, XAL); $1 \mathrm{~km} \mathrm{~S}$ of Agustín Melgar, on way to Río Solosúchil, $17^{\circ} 15^{\prime}$ N, 943' W, M. Nee y K. Taylor 29789 (F, XAL); vicinity of Campamento La Laguna, $17^{\circ} 17^{\prime} \mathrm{N}, 94^{\circ} 30^{\prime} \mathrm{W}$, M. Nee 29846 (F, NY); brecha La Laguna a El Elefante, $17^{\circ} 17^{\prime} \mathrm{N}, 94^{\circ} 30^{\prime} \mathrm{W}$, M. Vázquez 850 (XAL); $5 \mathrm{~km}$ al NW del campamento Hermanos Cedillo por la brecha Cedillo-La Escuadra, $17^{\circ} 16^{\prime} \mathrm{N}$, 94³6' W, M. Vázquez et al. 1671 (ENCB, F, MEXU, XAL). Mpio. Huatusco. Naranjas, 19¹2' N, 96 55' W, F. Ventura A. 5217 (ENCB, MICH). Mpio. Jalapa. on old Jalapa-Coatepec road, just S of Botanic Garden, 1.1 miles $\mathrm{N}$ of jct. with new Coatepec road, 19³0' N, 935' W, D. M. Kearns 589 (MEXU). Mpio. Minatitlán. $8.2 \mathrm{~km}$ al $\mathrm{N}$ de la terracería La Laguna-Río Grande, sobre el camino nuevo (no completo) a Belisario Domínguez, el cual sale de la terracería $14.7 \mathrm{~km}$ al E de La Laguna, $17^{\circ} 21^{\prime} \mathrm{N}, 9^{\circ} 22^{\prime} \mathrm{W}, T$. Wendt, A. Villalobos e I. Navarrete 2519 , 12/7/1980, (MEXU); zona de Uxpanapa, $13.5 \mathrm{~km}$ E of La Laguna, then $6.5 \mathrm{~km} \mathrm{~N}$ on dirty road to Belisario Dominguez, $12^{\circ} 20^{\prime} \mathrm{N}, 94^{\circ} 23^{\prime} \mathrm{W}, W$. Thomas, T. Wendt, $J$. Grimes y J. García 3551 (K, MICH, NY); $7.6 \mathrm{~km}$ al N de Poblado 10 en el camino a Hidalgo Amajac $17^{\circ} 22^{\prime} \mathrm{N}, 9^{\circ} 27^{\prime} \mathrm{W}, T$. Wendt, A. Villalobos e I. Navarrete 3980, 26/9/1992, (ENCB, MEXU). Mpio. Misantla. Misantla, 1955' N, 96 52' W, F. Ventura A. 8115 (CHAPA, ENCB, MEXU, MICH). Mpio. Pajapan. $5 \mathrm{~km}$ NW of Pajapan, SE slopes of Cerro San Martín Pajapan, 18¹7'30" N, 9443' W, M. Nee y J. I. Calzada 22782, (F, XAL). Mpio. San Andrés Tuxtla. Estación de Biología Tropical Los Tuxtlas, $18^{\circ} 34^{\prime}, 18^{\circ} 36^{\prime} \mathrm{N}, 95^{\circ} 04^{\prime}, 9^{\circ} 09^{\prime} \mathrm{W}$, J. I. Calzada 526 (F); ibid., R. Hernández y R. Cedillo 1262 (F, NY, XAL); ibid., G. Ibarra 2442 (CHAPA, XAL), 2460, (CHAPA), 2881 (CHAPA, MEXU); ibid., G. Martínez-Calderón 3095 (F, XAL); ibid., G. L. Webster y W. S. Armbruster 20978 (MEXU); Laguna Encantada, a 5 km al NE de San Andrés Tuxtla, por La Antigua, carretera a Mastagaga, $18^{\circ} 28^{\prime} \mathrm{N}, 95^{\circ} 10^{\prime} \mathrm{W}$, J. I. Calzada 10721 (XAL);

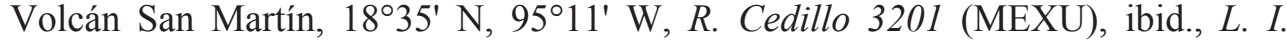
Nevling y A. Gómez-Pompa 2517 (F, NY). Municipio desconocido. km 6 del camino Cedillo-Río Alegre, Jesús Carranza, 17²10' N, 94²40' W, J. Dorantes 3934 (MEXU, MO, XAL); al E de Tecolapan, Los Tuxtlas, M. Sousa 2612 (MEXU).

Sicydium tamnifolium (H.B.K.) Cogn., DC. Monogr. Phan. 3: 905. 1881. Fevillea tamnifolia H.B.K., Nov. Gen. Sp. Pl. 7: 175. 1825. Tipo: Colombia, Guarumbo, Río Magdalena, A. Bonpland 1674 (holotipo P!; foto XAL!). Triceratia bryonioides A. Rich., In: Sagra, Hist. Nat. Cuba, II, 10: 299. 1845. 
Plantas herbáceas, rastreras a trepadoras, principalmente con tricomas articulado-septados glandulares, de 0.5 a $3 \mathrm{~mm}$ de largo, con frecuencia presentando tonalidades amarillentas o blanquecinas al secar; tallos delgados, sulcados, densa a esparcidamente glandular-pubescentes o glandular-puberulentos; láminas foliares ovado-cordadas, de 3 a $9.5 \mathrm{~cm}$ de largo y 2.5 a $9 \mathrm{~cm}$ de ancho, enteras a 3-angulosolobadas, ocasionalmente algunas hojas jóvenes hastadas, la base cordada a rectangular, los bordes enteros, el ápice agudo a acuminado, de consistencia herbáceo-cartácea, verdes a amarillento-verdosas o blanquecino-verdosas al secar, ambas superficies por lo común densa y suavemente pubescentes a puberulentas (rara vez casi glabras), los tricomas cortos y largos no glandulares generalmente adpresos y algunos pelos glandulares patentes esparcidos; zarcillos esparcida y diminutamente glandular-puberulentos, pecíolos de 1.3 a $6.7 \mathrm{~cm}$ de largo, sulcados, indumentados como el tallo; panículas estaminadas de (3-) 6 a 12 (-16) cm de largo, densamente glandular-pubescentes a glandular-puberulentas, brácteas en las axilas ovado-cordadas, ovado-lanceoladas o, las más pequeñas, angostamente lanceoladas, pedicelos filiformes, de $1.5 \mathrm{a} 4 \mathrm{~mm}$ de largo, glandular-puberulentos, articulados hacia la base, receptáculo de 0.3 a 0.4 de largo y $2 \mathrm{~mm}$ de ancho, pubescente, sépalos triangular-dentiformes, de $0.3 \mathrm{~mm}$ de largo, densamente pubescentes, pétalos linearlanceolados, de 0.9 a $1.5 \mathrm{~mm}$ de largo y 0.5 a $0.8 \mathrm{~mm}$ de ancho, agudos a acuminados, esparcidamente pubescentes a puberulentos, por lo común ligeramente fimbriados o erosos, filamentos nulos a casi tan largos como las anteras, éstas de 0.1 a $0.2 \mathrm{~mm}$ de largo, el conectivo nulo o muy escasamente desarrollado; panículas pistiladas de 10 a $12 \mathrm{~cm}$ de largo, pedicelos de 1 a $3 \mathrm{~mm}$ de largo, pubescentes, ovario ovoide, densamente pubescente, estilos y estigmas como en el género, estaminodios 3, por lo general presentes, algunas veces con el aspecto de estambres normales y funcionales; frutos globosos, de 4 a $8 \mathrm{~mm}$ de diámetro, densa a esparcidamente glandular-pubescentes, pedúnculos robustos, de 4 a $11 \mathrm{~mm}$ de largo, densamente glandular-puberulentos; semilla de 3 a $4 \mathrm{~mm}$ de diámetro.

Distribución y hábitat. Especie ampliamente distribuida en América tropical, desde México hasta Perú y también en las Antillas. Probablemente sea la que ocupa una mayor diversidad de hábitats, pues crece en un amplio intervalo altitudinal que va desde el nivel del mar hasta cerca de los 1700 m s.n.m., en bosques tropicales caducifolios, perennifolios y muy diversas asociaciones de vegetación secundaria. También se le ha recolectado en ambientes con vegetación riparia, en potreros, en zonas inundables y como ruderal o como arvense en milpas.

Nombres comunes y usos. En Oaxaca recibe la denominación "gin man" posiblemente de origen chinanteco pero de significado desconocido; en la Península de Yucatán (y también en algunas zonas de Guatemala) se le denomina "chak mots", un nombre maya que hace clara alusión a las características de sus raíces, pues 
se traduce como raíz delgada roja; en Querétaro y San Luis Potosí se ha registrado con el término "aisaan an wako", aparentemente de origen huasteco aunque de significado desconocido; en Tabasco recibe los nombres "sandía de culebra" y "sandía de rata"; finalmente, en Belice se le llama "bastard grape" y "wild grape". Aparentemente el único uso que se conoce para esta especie es el registrado para la Península de Yucatán, en donde sus raíces se emplean para la curación de quemaduras y llagas (Lira, 1988; Lira y Caballero, 2002).

Notas. Esta especie es sumamente variable en la pubescencia de las láminas foliares y otras partes vegetativas, las cuales pueden ser desde diminuta e inconspicuamente glandular-puberulentas, hasta densamente glandular-pubescentes. Como ejemplo de los dos extremos de esta variación están, por una parte, todos los materiales de la Península de Yucatán, cuyas hojas y tallos presentan un indumento más denso y, por otra, algunos materiales procedentes de los municipios de Huixtla y Tapachula en el sur de Chiapas, muchos de los cuales son casi glabros o al menos muy diminutamente puberulentos. No obstante, el hecho de presentar pedicelos de las flores estaminadas articulados hacia la base y estambres con los filamentos casi nulos o del mismo tamaño que las anteras, permiten distinguirla de las restantes especies del género presentes en México.

Ejemplares examinados. CAMPECHE: Mpio. Cd. del Carmen. $29 \mathrm{kms}$ al E de Francisco Escárcega, sobre la carretera 186 en el tramo Escárcega a Chetumal, E. Cabrera y H. de Cabrera 12564 (MEXU); $4 \mathrm{~km}$ al SE de Sabancuy, sobre la carretera Escárcega a $\mathrm{Cd}$. del Carmen, $18^{\circ} 57^{\prime} \mathrm{N}, 91^{\circ} 08^{\prime} \mathrm{W}$, E. Cabrera y $H$. de Cabrera 12696 (MEXU); Ejido Ojo de Agua, Rancho Palo Limón, km 231.5

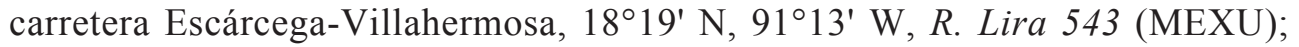
km 21 de la carretera Escárcega-Chetumal, R. Lira y J. S. Flores 569 (MEXU). Mpio. Champotón. Along Hwy 180, 7.9 miles N of Puente Champotón, just S of Cd. del Sol, $19^{\circ} 29^{\prime}$ N, 90²' W, D. M. Kearns y E. Martinez 564 (MEXU); along Hwy 261, ca. 6.5 miles S of Champotón, $19^{\circ} 19^{\prime}$ N, 9044' W, D. M. Kearns 575, 585 (MEXU); Si-Ho Playa carretera Champotón-Campeche, primer km adelante de hotel, 19³3' N, 9042' W, R. Lira y J. S. Flores 587, R. Lira 604 (MEXU); vicinity of Haltunchen, $14 \mathrm{~km} \mathrm{~N}$ of Champotón, 19³0' N, 90 $42^{\prime} \mathrm{W}, M$. Nee y T. C. Andres 32377 (NY, XAL); Champotón, 19³4' N, 99¹4' W, W. C. Steere 1747 (MICH, NY). Mpio. Hopelchén. Grutas de Calcehtok, 20³2' N, 8954' W, J. I. Calzada et al. 6679 (MEXU, XAL); ibid., S. Darwin et al. 2141 (F, MO, MEXU, NO); Hopelchen, 1945' N, 8951' W, M. Nee 32399 (NY, XAL); ejido Beljá, $19^{\circ} 00^{\prime} \mathrm{N}, 8^{\circ} 25^{\prime} \mathrm{W}$, E. Ucán, L. M. Ortega y J. Tun 6478 (MEXU). Mpio. Palizada. Rancho Chimali, km 10 de la carretera a Palizada, R. Lira 559 (MEXU; Canasayab, $19^{\circ} 18^{\prime} \mathrm{N}, 90^{\circ} 34^{\prime}$ W, C. L. Lundell 1411 (F, MICH); Palizada, $18^{\circ} 15^{\prime}$ N, 
92 $05^{\prime}$ W, E. Matuda 3829 (F, GH, MEXU, MICH, NY). Municipio desconocido. En los alrededores de la zona arqueológica de Edzná, a $20 \mathrm{~km}$ al S del km. 44 de la carretera Campeche-Hopelchén, 19³4' N, 99¹4' W, E. Cabrera y H. de Cabrera 7114 (MEXU); Campeche, sin datos específicos de localidad, J. Linden 986 (MICH). CHIAPAS: Mpio. Bejuco de Ocampo. $18 \mathrm{~km}$ al SE de Frontera Comalapa, carretera Motozintla-Comitán, $15^{\circ} 29^{\prime} \mathrm{N}, 92^{\circ} 07^{\prime} \mathrm{W}$, R. Lira et al. 1008 (MEXU). Mpio. Catzajá. Along shore of Laguna Catazaja, near El Cuyo, D. E. Breedlove 47257 (MEXU). Mpio. Huixtla. 6-8 km NE of Huixtla along road to Motozintla, $15^{\circ} 11^{\prime} \mathrm{N}, 92^{\circ} 26^{\prime} \mathrm{W}$, D. E. Breedlove 28552 (ENCB, MEXU); $12 \mathrm{~km}$ al NE de Huixtla, carretera a Motozintla, $15^{\circ} 29^{\prime} \mathrm{N}, 92^{\circ} 07^{\prime} \mathrm{W}$, R. Lira et al. 968 (MEXU). Mpio. Ixcomitán. $2 \mathrm{~km}$ al E de Ixcomitán, camino a Villa Hermosa, $E$. Martínez 3156 (MEXU); $8 \mathrm{~km}$ al S de Ixtacomitán, en La Lupita, O. Téllez 7803 (MEXU). Mpio. Mapastepec. $10 \mathrm{~km} \mathrm{SE} \mathrm{of} \mathrm{Mapastepec,} \mathrm{D.} \mathrm{E.} \mathrm{Breedlove} \mathrm{y} \mathrm{R.}$ F. Thorne 30676 (MEXU, NY); aproximadamente $3 \mathrm{~km}$ sobre el camino a la torre de microondas Mapastepec, desviación aprox. $6.5 \mathrm{kms}$ al ESE de Mapastepec, carretera Tonalá-Tapachula, $15^{\circ} 25^{\prime} \mathrm{N}, 92^{\circ} 47.5^{\prime} \mathrm{W}, R$. Lira y A. Reyes 1299 (MEXU). Mpio. Ocosingo. En zona Marqués de Comillas, $12 \mathrm{~km}$ al E de Pico de Oro camino al ejido Benemérito de las Américas, cerca del pozo Cantil, E. Martínez 16612 (MEXU); ejido Chajul, 160' N, 9056' W, E. Martínez, G. Domínguez y R. Lombera 26046 (MEXU). Mpio. Ocozocuautla de Espinoza. Southwest side of the Presa de Mal Paso, A. Shilom Ton 3776 (ENCB, LL, MEXU, MICH). Mpio. Tapachula. Near bridge of Hwy. Mex. 190, over Río Huixtla, 8 km (by air) NE of Huixtla, $15^{\circ} 11^{\prime} \mathrm{N}, 92^{\circ} 95^{\prime} \mathrm{W}, T$. C. Andres y M. Nee 145 (MEXU). Municipio desconocido. Downtown Comalapan, just SE of jct Ave. Central and 3A Calle Sur Poniente, D. M. Kearns y E. Martínez 557 (MEXU); Chiapas, sin datos específicos de localidad, J. Chavelas Polito 1676 (ENCB). OAXACA: Mpio. Chiltepec. 3-4 km al N de Chiltepec, sobre el camino a Santa Úrsula, $17^{\circ} 58^{\prime} \mathrm{N}, 96^{\circ} 13^{\prime} \mathrm{W}, R$. Lira y J. C. Soto 1178 (MEXU); Chiltepec and vicinity, $17^{\circ} 56^{\prime} \mathrm{N}, 96^{\circ} 12^{\prime} \mathrm{W}, G$. Martínez-Calderón 187 (GH, US), 173, 211 (MEXU). Mpio. San Lucas Ojitlán. Vista Hermosa, $18^{\circ} 02^{\prime} \mathrm{N}, 96^{\circ} 27^{\prime} \mathrm{W}$, J. I. Calzada 14210 (MEXU); $22 \mathrm{~km}$ al SW de San Lucas Ojitlán, carretera a San Lucas Ojitlán y Jalapa de Díaz, aproximadamente $7 \mathrm{~km}$ antes de llegar a la ranchería Los Ideales, $18^{\circ} 03^{\prime} \mathrm{N}, 96^{\circ} 18^{\prime} \mathrm{W}$, R. Lira y J. C. Soto 1165 (MEXU). Mpio. Soyaltepec. Cerro Verde al SE de Temascal, $18^{\circ} 13^{\prime} \mathrm{N}, 9^{\circ} 23^{\prime} \mathrm{W}$, L. Cortés y G. Cortés 1218 (MEXU). Municipio desconocido. Presa Temascal, camino a los vertederos, $18^{\circ} 13^{\prime} \mathrm{N}, 96^{\circ} 23^{\prime} \mathrm{W}, L$. Cortés y R. Torres 33 (MEXU); Parque Nacional de Chacahua, Distrito de Juquila, $R$. Cedillo y $R$. Torres 1509 (CHAPA, MEXU). PUEBLA: Mpio. Tlacotepec de Díaz. Alrededores de Tlacotepec, $18^{\circ} 29^{\prime} \mathrm{N}, 96^{\circ} 54^{\prime} \mathrm{W}$, P. Tenorio 15915 (MEXU). QUERÉTARO: Mpio. Jalpan. Al noroeste de Tanchanaquito, Los Sarros, L. López 417 (IEB); al N de Tanchananquito, La Barranquita, L. López 686 (IEB, MEXU); 
al N de Tanchananquito, entre La Barranquita y La Barranca Grande, L. López 716 (IEB, MEXU); 8-10 km al N de Carrizal, Isla del Río, B. Servín 392 (IEB, MEXU). Mpio. Landa de Matamoros. Río Tancuilín, al W de Neblinas, E. Carranza y E. Pérez 5196 (CHAPA, IEB); al SE de Tangojó, sobre el Río Moctezuma, E. Carranza y E. Pérez 5647 (IEB, IZTA); 4 km al E de Tangojó, R. Fernández Nava 4253 (ENCB); aproximadamente $10.5 \mathrm{~km}$ al SE de Agua Zarca, sobre el camino a Pisaflores, $R$. Lira et al. 1356, 1356 (IEB); $11 \mathrm{~km}$ al SE de Agua Zarca, sobre el camino a Pisaflores, J. Rzedowski 42798 (IEB, MEXU); $10 \mathrm{~km}$ al SE de Agua Zarca, sobre el camino a Pisaflores, J. Rzedowski 45176 (IEB, MEXU). QUINTANA ROO: Mpio. Cozumel. Cobá, C. L. Lundell y A. Lundell 7682 (F, GH, LL/TEX, MICH, US). Mpio. Felipe Carrillo Puerto. km 115 carretera Carrillo Puerto-Valladolid, $20^{\circ} 29^{\prime} \mathrm{N}, 8^{\circ} 16^{\prime} \mathrm{W}, R$. Lira y E. Ucán 636 (CICY, MEXU, XAL). Mpio. José María Morelos. Chichankanab, G. F. Gaumer 2059 (BR, F, MICH, NY), 2064 (F, NY). Mpio. Othón P. Blanco. Laguna Bacalar, C. Chan $y$ M. Burgos 1400 (XAL). SAN LUIS POTOSÍ: Mpio. Ciudad Valles. $2 \mathrm{~km}$ of El Abra on the road from Cd. Valles to Tampico, 21 ${ }^{\circ} 58^{\prime} \mathrm{N}, 98^{\circ} 54^{\prime} \mathrm{W}, B$. Bartholemew 3490 (MEXU, NY); rancho Pago Pago, 3.5 miles by road W of Chontal, near juncture of Río Mesillas and Río Mico, P. A. Fryxell y W. R. Anderson 3481 (CHAPA, ENCB, MEXU, MICH). Mpio. San Antonio. Tanjasmec, J. B. Alcorn 1960 (TEX). Mpio. Tamazunchale. Alrededores de Tamán, 21ํํ' N, 98 53' W, J. Rzedowski 11028 (ENCB). Mpio. Xilitla. 4 km al W de la 'Y Griega' de Xilitla, $P$. Tenorio y C. Romero 565 (K, MEXU). Municipio desconocido. $21.4 \mathrm{~km}$ of Ciudad Valles on the road to Río Verde, B. Bartholemew et al. 3520 (MEXU); hwy 80 from Cd. Valles to S.L.P., just inside S.L.P. near the village of El Sabinito, km 40, A. C. Gibson y L. C. Gibson 2657 (ENCB); hwy 120, 1.9 miles SW of jct Hwy 85, D.M. Kearns 483 (MEXU); Tamasopo Canyon, 215' N, 99²4' W, C. G. Pringle 3093 (BR, F, GH, MEXU, MICH, MO, NY, US); San Luis Potosí, sin datos específicos de localidad, $20^{\circ} 10^{\prime} \mathrm{N}, 100^{\circ} 59^{\prime} \mathrm{W}$, C. G. Pringle $5106(\mathrm{GH})$ : $25 \mathrm{~km}$ al $\mathrm{W}$ de $\mathrm{Cd}$. Valles, sobre la carretera a Río Verde, $21^{\circ} 57^{\prime} \mathrm{N}, 99^{\circ} 12^{\prime} \mathrm{W}$, J. Rzedowski 24381 (F, MICH, TEX). TABASCO: Mpio. Balancán. Balancán, $17^{\circ} 48^{\prime} \mathrm{N}, 9^{\circ} 32^{\prime} \mathrm{W}$, A. Guadarrama 489 (MEXU); cerca del poblado El Triunfo,

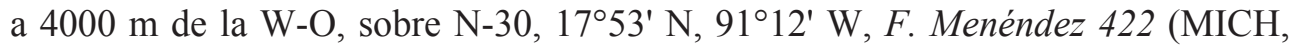
XAL). Mpio. Centla. Laguna El Horizonte, en el km 30 de la carretera Villa Hermosa-Frontera, M. A. Magaña y A. Guadarrama 2213 (MEXU). Mpio. Centro. Km 13 carretera Villahermosa a Macuspana, Dos Montes, $17^{\circ} 58^{\prime} \mathrm{N}$, 9248' W, L. A. González y L. A. Pérez P556 (ENCB, MICH, XAL); a 13 km de Villahermosa, por la carretera a Escárcega, Cam. y a unos $800 \mathrm{~m}$ al SE, $17^{\circ} 58^{\prime} \mathrm{N}$, $92^{\circ} 48^{\prime}$ W, L. A. González y L. A. Pérez 4159 (ENCB). Mpio. Comalcalco. Villa Aldama, 18¹4' N, 9321' W, F. Ventura A. 21332 (NY). Mpio. Huimanguillo. El 33, F. Ventura A. 20449 (ENCB, XAL). Mpio. Nacajuca. Guatacala, 18¹0' N, 
92 59' W, R. Ortega y W. Márquez O-924 (XAL). Mpio. Tlacotalpa. $9.3 \mathrm{~km}$ antes (N) del Ej. Zunu y Patastal, camino de Tlacotalpa hacia Tapijulapa, C. P. Cowan et al. 3450 (MEXU). Mpio. Teapa. Al S de Los Azufres, L. Rico y E. Martínez 51, 720 (MEXU, NY); along the bank of the Río Teapa, $2 \mathrm{~km} \mathrm{SE} \mathrm{of} \mathrm{Teapa,}$ 17³4' N, 92 57' W, G. Davidse et al. 29553 (MEXU, MO). TAMAULIPAS: Mpio. Gómez Farías. 3 km N of Gómez Farías, P. A. Fryxell 3767 (CHAPA, ENCB, F, NY). Municipio desconocido. Galeana cañon, L. A. Kenoyer 3493 (GH); 55 mi. $\mathrm{S}$ of Cd. Victoria, $23^{\circ} 02^{\prime} \mathrm{N}, 9^{\circ} 05^{\prime} \mathrm{W}, F$. G. Meyer y D. J. Rogers 2858 (BR, MO). VERACRUZ: Mpio. Actopan. Camino a Cansa Burros, $2 \mathrm{~km}$ al $\mathrm{N}$ de Paso del Cedro, 1932' N, 96²3' W, R. Acosta y F. Vázquez 686 (XAL); Estación Biológica El Morro de La Mancha, 19³6' N, 96²2' W, B. Guerrero 2210 (XAL); Chapopote, 19³0' N, 96³7' W, F. Ventura A. 9295 (CHAPA, MEXU, MICH). Mpio. Chicontepec. En el poblado de Tlacolula, $21^{\circ} 06^{\prime} \mathrm{N}, 97^{\circ} 58^{\prime} \mathrm{W}$, J. I. Calzada 5609 (CHAPA, ENCB, F, XAL), 5628 (F, XAL). Mpio. Coatepec. 3 km E de Tuzamapan, 19²5' N, 9652' W, M. Vázquez 2198 (ENCB, F, MEXU, NY, XAL). Mpio. Coazintla. Palmar de Zapata, M. E. Cortés 222 (MEXU). Mpio. Cosamaloapan. West side of Río Papaloapan, $3 \mathrm{~km} \mathrm{~S}$ of Cosamaloapan, 18 ${ }^{\circ} 19^{\prime} \mathrm{N}$, 9549' W, M. Nee y K. Taylor 29211 (F, NY, XAL). Mpio. Dos Ríos. Plan del Río, 185' N, 9702' W, F. Ventura A. 4349 (ENCB, MICH). Mpio. Martínez de la Torre. Malnapan, $20^{\circ} 04^{\prime} \mathrm{N}, 9^{\circ} 04^{\prime} \mathrm{W}, F$. Ventura A. 12637 (XAL), 14544 (CHAPA, XAL). Mpio. Misantla. Misantla, 195 $55^{\prime} \mathrm{N}, 96^{\circ} 52^{\prime} \mathrm{W}$, C. A. Purpus 5973 (F, GH, MO, NY), 5978 (P). Mpio. Pánuco. Tramo carretera Pánuco-Miradores o Tanchikui, $7 \mathrm{~km}$ al $\mathrm{O}$ de Pánuco, $22^{\circ} 03^{\prime} \mathrm{N}, 98^{\circ} 14^{\prime} \mathrm{W}$, C. Gutiérrez y L. Guerrero 1976 (MEXU, XAL). Mpio. Papantla. $2.5 \mathrm{~km}$ E of entrance to ruins of El Tajín, 4 km SW of Papantla, 20²6' N, 97²1' W, M. Nee y G. Diggs 24639 (F, NY). Mpio. Puente Nacional. Barranca de Palmillas, $2 \mathrm{~km}$ al SE de dicha población, $19^{\circ} 12^{\prime}$ N, 965' W, M. E. Medina y F. Vázquez 366 (MEXU, XAL). Mpio. San Andrés Tuxtla. Laguna Encantada, cerca $5 \mathrm{~km}$ NE de San Andrés Tuxtla, $18^{\circ} 27^{\prime} \mathrm{N}$, 9509' W, J. H. Beaman 5247 (F, MEXU, MO, NY, XAL), 5254 (ENCB, F, MEXU, NY, XAL); Salto de Eyipantla, $5 \mathrm{~km}$ (by air) S of San Andrés Tuxtla, $18^{\circ} 23.5^{\prime} \mathrm{N}, 95^{\circ} 12.5^{\prime} \mathrm{W}$, M. Nee 23637 (F, NY, XAL); Cebollal, F. Ponce 128 (MEXU). Mpio. San Lorenzo Tenochtitlán. Atrás del campamento, 1949' N, 9356' W, J. Chavelas Polito ES-2797 (MEXU). Mpio. Santiago Tuxtla. Santiago Tuxtla, $18^{\circ} 28^{\prime} \mathrm{N}, 95^{\circ} 18^{\prime} \mathrm{W}$, G. Martínez-Calderón 1490 (F, GH, MEXU, MICH, MO). Mpio. Tantoyuca. Wartenberg near Tantoyuca, prov. Huasteca, L. C. Ervendberg 258 (GH). Mpio. Tecolutla. Rancho Palo Hueco, 20²9' N, 9700' W, R. Rodríguez 51 (XAL). Mpio. Tepetzintla. San José de Copaltitla, $7 \mathrm{~km}$ al NE de Tepetzintla, $21^{\circ} 12^{\prime}$ N, 9752' W, G. Castillo y A. Benavides 2311 (F, XAL), L. Tapia y F. Vázquez 121 (XAL). Mpio. Tlaltetela. Puente Río Pescado, F. Ventura A. 11811 (ENCB, MEXU, MICH); El Limón, F. Ventura A. 14085 
(XAL). Mpio. Tlapacoyan. Rancho El Relicario, $20^{\circ} 58^{\prime} \mathrm{N}, 97^{\circ} 13^{\prime} \mathrm{W}$, F. Ventura A. 697, 698 (ENCB, MICH). Mpio. Veracruz. Ranchería Nevería, carretera Antigua Nacional Xalapa-Veracruz, 1911' N, 96¹8' W, C. Gutiérrez 828 (XAL). Municipio desconocido. Cerro $11 \mathrm{~km}$ al S de Palma Sola, frente a Villa Rica, $J$. Dorantes y A. Calles 1176 (MEXU); along Hwy 145, 0.7 miles SE of La Granja, D. M. Kearns 582 (MEXU); Ignacio de la Llave, ejido Palmas Cuatas, G. MartínezCalderón 1547 (ENCB, MEXU, MICH, MO, XAL); Río Vista, Río Coatzacoalcos, $1.5 \mathrm{~km}$ de Jesús Carranza, $18^{\circ} 00^{\prime} \mathrm{N}, 9^{\circ} 29^{\prime} \mathrm{W}$, L. I. Nevling y A. Gómez-Pompa 2546 (F); Cerro Monte de Oro, C. Vázquez-Yanes 587 (MEXU). YUCATÁN: Mpio. Chablekal. Zona arqueológica de Dzibilchaltun, A. Bradburn y S. Darwin 1204 (CICY, F, MEXU, NO); ibid, R. Lira 415 (MEXU, XAL). Mpio. Dzoncauich. Chacmay, J. S. Flores 10095 (XAL). Mpio. Izamal. Izamal, 2059' N, 8901' W, G. F. Gaumer 922 (BR, F, GH, K, MO, NY, US), 932 (BR, MICH, MO, NY). Mpio. Mérida. Buenavista, G. F. Gaumer s. n. (F). Mpio. Oxkutzkab. Zona arqueológica de Xlapak, 20¹0' N; 89³7' W, R. Lira 405, J. J. Ortiz 282, (MEXU); huerto dentro de una casa de Oxkutzcab, R. Lira y P. Yam 781 (XAL). Mpio. Santa Elena. Zona arqueológica de Kabah, A. Puch y M. Narváez 530 (XAL). Mpio. Temozón. En la comunidad de Yokdzonot, a $42 \mathrm{~km}$ del municipio, J. A. Aguilar y S. Diez 34 (MEXU). Mpio. Tinúm. Chichén Itzá, W. C. Steere 1459 (F, MEXU, MICH, MO), 8587 (MEXU). Mpio. Valladolid. Pixoy, E. Ucán 4336 (XAL). Mpio. Yaxcabá. Kankabdzonot, G. F. Gaumer 23523 (NY), 23525 (F, US). Municipio desconocido. En los alrededores de la zona arqueológica de Mayapán, a $1 \mathrm{~km}$ al S Telchaquillo, carretera Tecoh-Oxkutzcab, E. Cabrera y H. de Cabrera 9123 (MEXU); San Anselmo, G. F. Gaumer 2058 (F, GH, MO, US); ruins of Aké 30

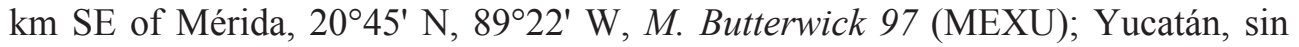
datos específicos de localidad, G. F. Gaumer 24311 (BR, F, GH, MEXU, MO, NY), J. Linden 987 (K), s. n. (P).

Sicydium tuerckheimii Donn. Sm., Bot. Gaz. 52: 49. 1911. Tipo: Guatemala, Alta Verapaz, Cubilquitz, H. Tuerckheim 1914 (holotipo US!). Fig. 5.

Plantas herbáceas, trepadoras, con tricomas articulado-septados, glandulares y no glandulares, de hasta $3 \mathrm{~mm}$ o más de largo, frecuentemente presentando una tonalidad pardo obscuro-rojiza en material de herbario; tallos delgados, sulcados, pubescentes a puberulentos, algunas veces glabrescentes con la edad; láminas foliares ovado-cordadas, de 5.5 a $16.5 \mathrm{~cm}$ de largo y 6 a $11 \mathrm{~cm}$ de ancho, enteras a raramente 3-anguloso-lobadas, la base cordada a rectangular, los bordes enteros, el ápice agudo a acuminado, de consistencia cartácea a coriácea, frecuentemente de color pardo-rojizo obscuro al secar, superficie adaxial puberulento-escabrosa y áspera, la abaxial densa y suavemente pubescente, pecíolos de 1.5 a $6 \mathrm{~cm}$ de largo, 


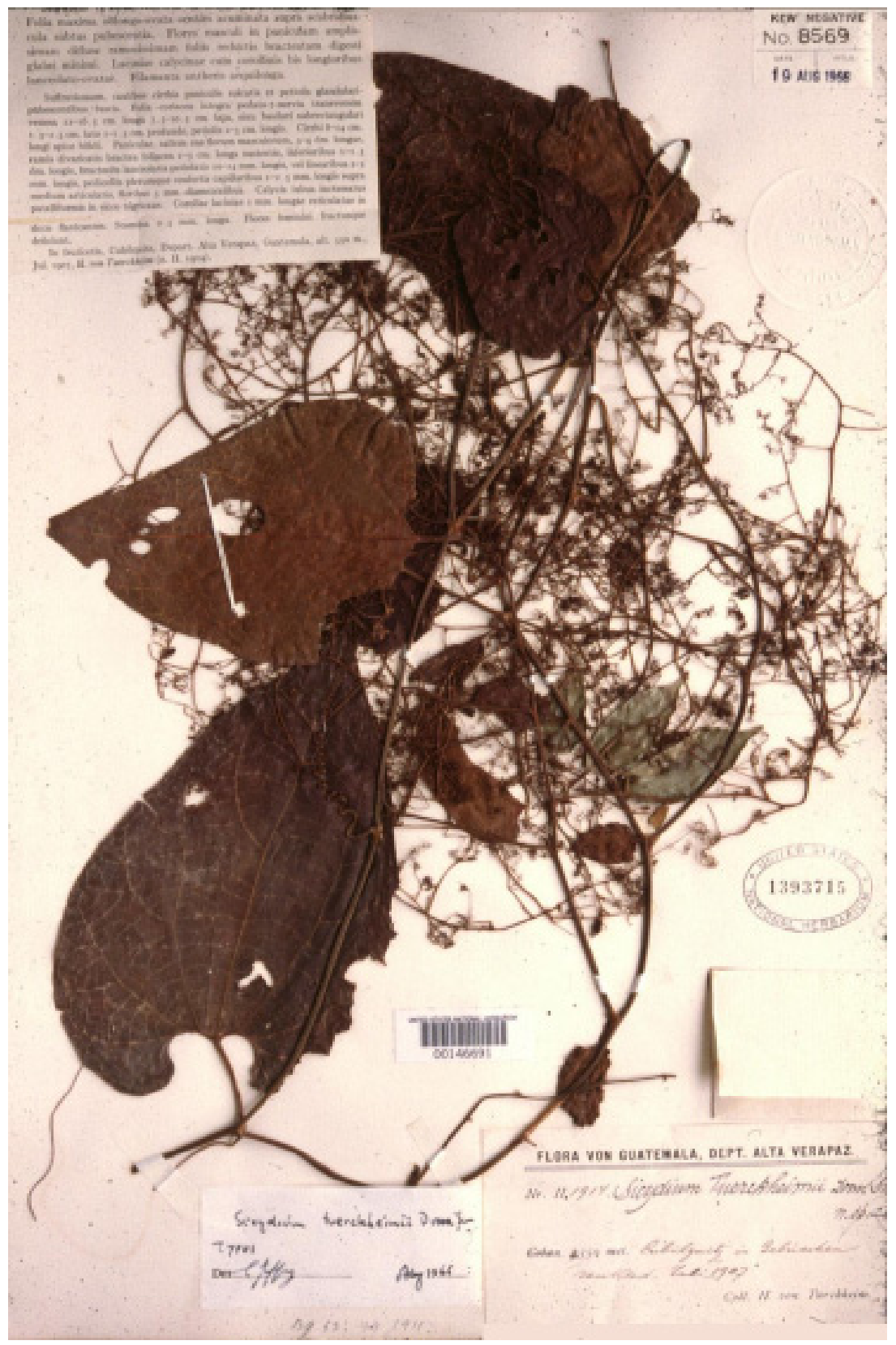

Fig. 5. Ejemplar tipo de Sicydium tuerckheimii Donn. Sm. 
sulcados, indumentados como el tallo; zarcillos esparcida y diminutamente puberulentos; panículas estaminadas de (10-) 20 a $40 \mathrm{~cm}$ de largo, pubescentes a puberulentas, brácteas ovado-lanceoladas a lanceoladas, pedicelos filiformes, de $1 \mathrm{a} 4 \mathrm{~mm}$ de largo, puberulentos, glabrescentes, articulados a la mitad o poco más arriba, receptáculo de 0.4 a $0.5 \mathrm{~mm}$ de largo y 0.5 a $0.7 \mathrm{~mm}$ de ancho, puberulento, sépalos triangulares, de 0.5 a $0.6 \mathrm{~mm}$ de largo y $0.3 \mathrm{~mm}$ de ancho, puberulentos, pétalos ovado-lanceolados, de 0.9 a $1.5 \mathrm{~mm}$ de largo y 0.5 a $0.8 \mathrm{~mm}$ de ancho, agudos a acuminados, puberulentos, bordes enteros, filamentos de 0.4 a $0.6 \mathrm{~mm}$ de largo, anteras de 0.2 a $0.3 \mathrm{~mm}$ de largo, conectivo comparativamente ensanchado y ligeramente bifurcado en el ápice; panículas pistiladas de 12 a $13(-20) \mathrm{cm}$ de largo, pedicelos robustos, de 1 a $3 \mathrm{~mm}$ de largo, puberulentos, ovario ovoide, densamente pubescente a tomentoso, perianto como en las estaminadas, pero el receptáculo muy reducido a obsoleto, estaminodios no vistos; frutos globosos, de 8 a $12 \mathrm{~mm}$ de diámetro, pubescentes, glabrescentes con la edad, pedúnculos robustos, de 2 a $4 \mathrm{~mm}$ de largo, puberulentos; semilla de 4 a $6 \mathrm{~mm}$ de diámetro.

Distribución y hábitat. $S$. tuerckheimii Esta especie se distribuye de México a Centroamérica cuando menos. Crece en bosques tropicales perennifolios, subperennifolios, mesófilos de montaña y en vegetación secundaria derivada de ellos, entre 10 y 1400 m s.n.m.

Notas. Aunque algunos ejemplares de esta especie con escasa pubescencia en la superficie abaxial de las láminas foliares pudieran identificarse como extremos de la variación de Sicydium schiedeanum, existe una diferencia importante en las dimensiones de sus granos de polen, siendo mayores los de S. tuerckheimii, los cuales, de hecho, están entre los más grandes del género (Lira et al., 1998).

Ejemplares examinados. CHIAPAS: Mpio. Motozintla de Mendoza. Cerro del Boquerón, C. A. Purpus 7400 (F). Mpio. Ocosingo. 15 km NW de Boca Lacantum, camino a Palenque, E. Martínez y M. A. Soto 18611 (MEXU). OAXACA: Mpio. Chiltepec. Chiltepec and vicinity, $17^{\circ} 56^{\prime} \mathrm{N}, 9^{\circ} 12^{\prime} \mathrm{W}$, G. Martínez-Calderón 102 (GH, MEXU), 463 (GH, MEXU, US), 736 (MICH). Mpio. Tuxtepec. Camino a los vertederos, a $4 \mathrm{~km}$ SE de Temazcal, Distrito de Tuxtepec, M. Sousa et al. 13083 (MEXU). PUEBLA: Mpio. Zapotitlán de Méndez. $6 \mathrm{~km}$ al NW de Zapotitlán, carretera a Tepango, $20^{\circ} 03^{\prime} \mathrm{N}, 97^{\circ} 45^{\prime} \mathrm{W}$, G. Toriz, A. Campos y O. Téllez 499 (MEXU). QUERÉTARO: Mpio. Landa de Matamoros. $6 \mathrm{~km}$ al NE de Agua Zarca, sobre el camino a Neblinas, J. Rzedowski 46535 (IEB, MEXU). TABASCO: Mpio. Cárdenas. San Juan Bautista, J. N. Rovirosa 24 (US); cerca del ingenio Benito Juárez, F. Ventura A. 19998 (ENCB, MEXU). VERACRUZ: Mpio. Vega de 
Alatorre. Sierra de Chichimecas al W de Santa Gertrudis, $19^{\circ} 52^{\prime} \mathrm{N}, 96^{\circ} 35^{\prime} \mathrm{W}, G$. Castillo y A. Benavides 2090 (XAL).

\section{AGRADECIMIENTOS}

Se agradece a los curadores de los herbarios citados en el trabajo por el préstamo de ejemplares y al Biól. José L. Alvarado, del Instituto Nacional de Antropología e Historia de México por su colaboración en los estudios palinológicos. Para la realización de este trabajo se contó con el apoyo económico de la Comisión Nacional para el Conocimiento y Uso de la Biodiversidad (CONABIO), a través del proyecto "Estado actual y fitogeografía de las especies de la familia Cucurbitaceae endémicas a México" (CONABIO Q-010), el cual estuvo a cargo del autor de 1998 a 2001.

\section{LITERATURA CITADA}

Cogniaux, A. 1881. Cucurbitacées. In: De Candolle, A. y C. De Candolle (eds.). Monogr. Phan. 3: 325-951.

Cogniaux, A. 1916. Cucurbitaceae-Fevilleae et Melothrieae. In: Engler, A. (ed.). Das Pflanzenreich IV. 275.1: 1-277.

Dieterle, J. V. A. 1976. Cucurbitaceae. Flora of Guatemala. Fieldiana Bot. 24(11): 306-395.

Jeffrey, C. 1990. Appendix. An outline classification of the Cucurbitaceae. In: Bates, D. M, R. W. Robinson y C. Jeffrey (eds.). Biology and utilization of the Cucurbitaceae. Cornell University Press. Comstock. pp. 449-463.

Jeffrey, C. y B. Trujillo. 1992. Cucurbitaceae. In: Morillo, G. (ed.). Flora de Venezuela. Fondo Editorial Acta Científica Venezolana. Caracas, Venezuela. pp.11-201.

Kearns, D. M. 1994. The genus Ibervillea (Cucurbitaceae): An enumeration of the species and two new combinations. Madroño 41: 13-22.

Lira, R. 1988. Cucurbitaceae de la Península de Yucatán: taxonomía y etnobotánica. Tesis de Maestría en Ciencias (Ecología y Recursos Bióticos). Instituto Nacional de Investigaciones sobre Recursos Bióticos. Xalapa, Veracruz. 329 pp.

Lira, R. 1995. A new species of Sicydium (Cucurbitaceae: Zanonioideae, Zanonieae, Sicydiinae) for the Flora Mesoamericana. Novon 5: 284-286.

Lira, R., J. L. Alvarado y M. Ayala-Nieto. 1998. Pollen morphology in Sicydium (Cucurbitaceae: Zanonioideae). Grana 37: 215-221.

Lira, R. y J. Caballero. 2002. Ethnobotany of the wild Mexican Cucurbitaceae. Econ. Bot. 56: 380-398.

Lira, R., J. L. Villaseñor y E. Ortiz. 2002. A proposal for the conservation of the family Cucurbitaceae in Mexico. Biodiversity and Conservation 11: 1699-1720. 
Nee, M. 1993. Cucurbitaceae. In: Sosa, V. (ed.). Flora de Veracruz. Fascículo 74. Instituto de Ecología A.C./University of Riverside. Xalapa, Ver. 133 pp.

Schlechtendal, D. F. L. 1832. De plantis mexicanis. Linnaea 7: 380-400.

Smith, J. D. 1911. Plants from Central America. Bot. Gaz. 52: 49-50.

Standley, P. C. y J. A. Steyermark. 1944. Studies of Central American plants. IV. Field Mus. Nat. Hist. Bot. Ser. 23: 31-109.

Wunderlin, R. P. 1978. Cucurbitaceae. Flora of Panama. Part IX. Ann. Missouri Bot. Gard. 65:285-368.

Recibido en agosto de 2002.

Aceptado en agosto de 2004. 